\title{
SPECTROSCOPIC OBSERVATIONS OF PLANETARY NEBULAE IN THE MAGELLANIC CLOUDS
}

\author{
M.W. FEAST \\ (Radcliffe Observatory, Pretoria, South Africa)
}

\begin{abstract}
A programme of spectroscopic observations of planetary nebulae in both Magellanic Clouds has been underway in Pretoria for some years, and some preliminary results for the LMC have been published (Feast, 1964b). More extensive observations are now available (altogether in both clouds 112 spectra of 39 objects), and these are being prepared for publication elsewhere. Below are summarized the main conclusions so far deduced.

The radial velocities depend very largely on $48 \AA / \mathrm{mm}$ plates and should be of good accuracy. Lower-dispersion spectra have less weight for velocities but are very valuable in that, in general, many of the fainter lines are observed and a better idea can be obtained of the physical nature of the object. For instance, in several objects high densities are indicated by the [OII] doublet, consistent with the classification of these objects as planetaries, and in one object a broad emission band (mainly CIII) from the central star was detected.
\end{abstract}

In the LMC there appears to be no significant systematic deviation of the planetary velocities from the rotation curve defined by extreme Population-I objects (supergiant stars, diffuse nebulae and interstellar gas) despite a recent suggestion of such a deviation (Webster, 1965). It has been a problem for some while that the centre of rotation of the $\mathrm{LMC}$ is displaced $1 \mathrm{kpc}$ from the optical centre. However, whereas the extreme Population-I objects are, in fact, distributed symmetrically about the centre of rotation, the planetaries have a centre of symmetry near the optical centre (Westerlund and Smith, 1964). Two alternative interpretations seem possible. Either, the centre of rotation is the real mass centre, in which case we must postulate high obscuration to the North of the optical bar (Wesselink, 1966), unless there are large quantities of undetected matter such as $\mathrm{H}_{2}$ in the LMC. Or, and this is an attractive possibility, we must adopt the view (advocated by de Vaucouleurs) that we are observing non-circular motions in a barred spiral. Whichever explanation is adopted, the present work indicates that the observed systematic velocity pattern applies to objects of a wide range of ages.

For the LMC the velocity dispersion of the planetaries corrected for observational error is $22 \pm 3$ (s.e.) $\mathrm{km} / \mathrm{sec}$ with no significant deviation from a gaussian distribution. This dispersion is significantly greater than for extreme Population-I objects $(9 \cdot 6 \pm 1 \cdot 1$

Osterbrock and O' Dell (eds.), Planetary Nebulae, 34-35. I.A.U. 
(s.e.) $\mathrm{km} / \mathrm{sec}$; Feast, 1964a) and may be taken as evidence for a collapse of the LMC to a plane (as in the case of the Galaxy). There is no large change in velocity dispersion of the planetaries from the centre to the edge of the LMC, in contrast to the results for the Galaxy. However, there is, of course, a much less marked central condensation of the planetaries in the LMC than in the Galaxy. An interesting indication has been found that the velocity dispersion of the planetaries increases with decreasing brightness. In addition there is some suggestion of a different surface distribution for bright and faint planetaries.

The radial velocities of planetary nebulae in the SMC show a clustering around two distinct values independent of position in the Cloud. These two groups are strongly reminiscent of the double peaks in the $21-\mathrm{cm}$ profiles. The results suggest the same overall flow pattern for the planetaries and the $\mathrm{HI}$ gas though there is no detailed correlation between the positions and velocities of the planetaries and the interstellar gas. Hindman (1967) has interpreted the $21-\mathrm{cm}$ results in terms of a number of large expanding shells in the SMC. However, this would require the planetaries to be young objects ( $<10^{7}$ years) and a detailed correlation of positions and velocities with extreme Population-I objects would then be expected. A promising approach appears to be motion in a barred spiral with the bar seen almost end on and with gas and stars flowing outwards from each end of the bar.

A distinct difference has been found between the two Clouds in the frequency of occurrence of planetaries of different excitation classes. Low-excitation planetaries predominate in the SMC. In this respect the Galaxy seems to be more similar to the LMC than to the SMC. In the LMC itself there is an indication that high-excitation planetaries are concentrated amongst the lower luminosity objects. Further study of this question should yield interesting results.

\section{References}

Feast, M.W. (1964a) Mon. Not. R. astr. Soc., 127, 195.

Feast, M.W. (1964b) Observatory, 84, 266.

Hindman, J. V. (1967) Austr. J. Phys., 20, 147.

Webster, L. (1965) in Symposium on the Magellanic Clouds, Ed. by J.V. Hindman and B.E. Westerlund, Canberra, p. 29.

Wesselink, A.J. (1966) Astr. J., 71, 185.

Westerlund, B. E., Smith, L.F. (1964) Mon Not. R. astr. Soc., 127, 449. 